\title{
FINANCE AND ENERGY. ANALYSIS OF THEIR RELATIONSHIP AND FUTURE DEVELOPMENT TRENDS
}

\author{
Francesco Zappia ${ }^{194}$ \\ Le Chen ${ }^{195}$ \\ Pietro Pavone ${ }^{196}$ \\ Roberta Pisani ${ }^{197}$
}

https://doi.org/10.31410/itema.2018.495

\begin{abstract}
Through an overview of recent developments in finance and in the energy sector, this work represents an analysis of the relationship of their development trends. This study is carried out through an interdisciplinary approach and we decided to prefer the observational research method in order to expand the people's perceptual knowledge in inspiring their thoughts and discovering new ways of development for finance and energy. From the original physical currency to banknotes then to electronic payment methods with the development of the internet economy, internet financial digital currency is becoming a new concept. Bitcoin was born in 2008 and since 2012 the Bitcoin's raised monetary value has allowed people to remember two major financial crises recently. It's still a fiat money (without intrinsic value) which is only as good as the Organization that issues it. Included but not limited to whether the entity is defaulting, the currency will become worthless. Because Bitcoin is not issued by any central bank, but by the information service provider which exists outside the monetary system, there is no centralized management of the country, and there is a great risk of losing control. Finance is not entirely a virtual economy. So ultimately, currency development trends must return to intrinsic value. For the foregoing, there is the possibility for the energy currency, not only for its uniform measurement but also for its good stability characteristics. As an energy currency, it is not necessary to print, not to apply the exchange rate, no corresponding pollution, and even better it will help to alleviate the environmental problem in the current phase and in a practical and effective way to face the challenges of global environmental degradation. The international unit of energy is joule and we can consider it as the unit of energy money because of energy and its intrinsic value. As stated by Brett Scott, there are good reasons to consider energy as a potential currency. If the future integrates energy into the financial system and becomes a means of payment, it will not only eliminate the risk of currency depreciation through a global unified measurement unit, unimpeded flow and easily exchange as a general equivalent, but also optimize energy saving and use to achieve the protection of environment and to realize the original nature of human return to nature. The emphasis is given to the possibility of integration of the financial sector with the energy one, a theme that can be framed between economics and scienceltechnology or their combination. The aim of this research is to develop a parametric numerical model able to foresee the possibility of combining finance and energy, allowing for both a sustainable development. This will be realized by reviewing all different approaches to the subject and all the solutions created. This will allow us to analyze the pros and cons of all the existing cryptocurrencies related to energy sector. The last step of this analysis consists in identifying the main characteristics that this cryptocurrency must have in order to achieve the desired results.
\end{abstract}

\footnotetext{
${ }^{194}$ E-Campus University (Novedrate, Italy), StratEgo TLC - Research Division (Milan, Italy)

195 E-Campus University (Novedrate, Italy), StratEgo TLC - Research Division (Milan, Italy)

${ }^{196}$ E-Campus University (Novedrate, Italy)

${ }^{197}$ StratEgo TLC - Research Division (Milan, Italy)
} 
Keywords: Cryptocurrency, development, integration, finance, energy, innovation.

\section{INTRODUCTION}

$\mathrm{W}$ ith the development of the internet economy, digital finance and cryptocurrecies are becoming new concepts. Their development is recording at a rate far beyond our expectations.

We can also feel that the internet technology is a revolution and it can be defined as a disruptive technology, subverting many traditional industries. How many physical stores have been subverted by Amazon and Taobao, and the people are already familiar with digital currency, which can drive economic development to a certain extent.

About digital currency, you have to mention Bitcoin. It's as a concept, was born in 2008 by a mysterious expert named "Zhong Ben Cong", at the same time as the US subprime mortgage crisis and even the global financial crisis.

At that time, Bitcoin was not worth the money, but because there were always players trading, some people began to publicize the hype, which gradually increased from 2009 to 2012, especially in 2012, showing explosive growth. This has made many players who were not Bitcoin come in and use this as a financial investment.

Figure 1: Bitcoin Price History Chart. Source: CoinMarketCap.

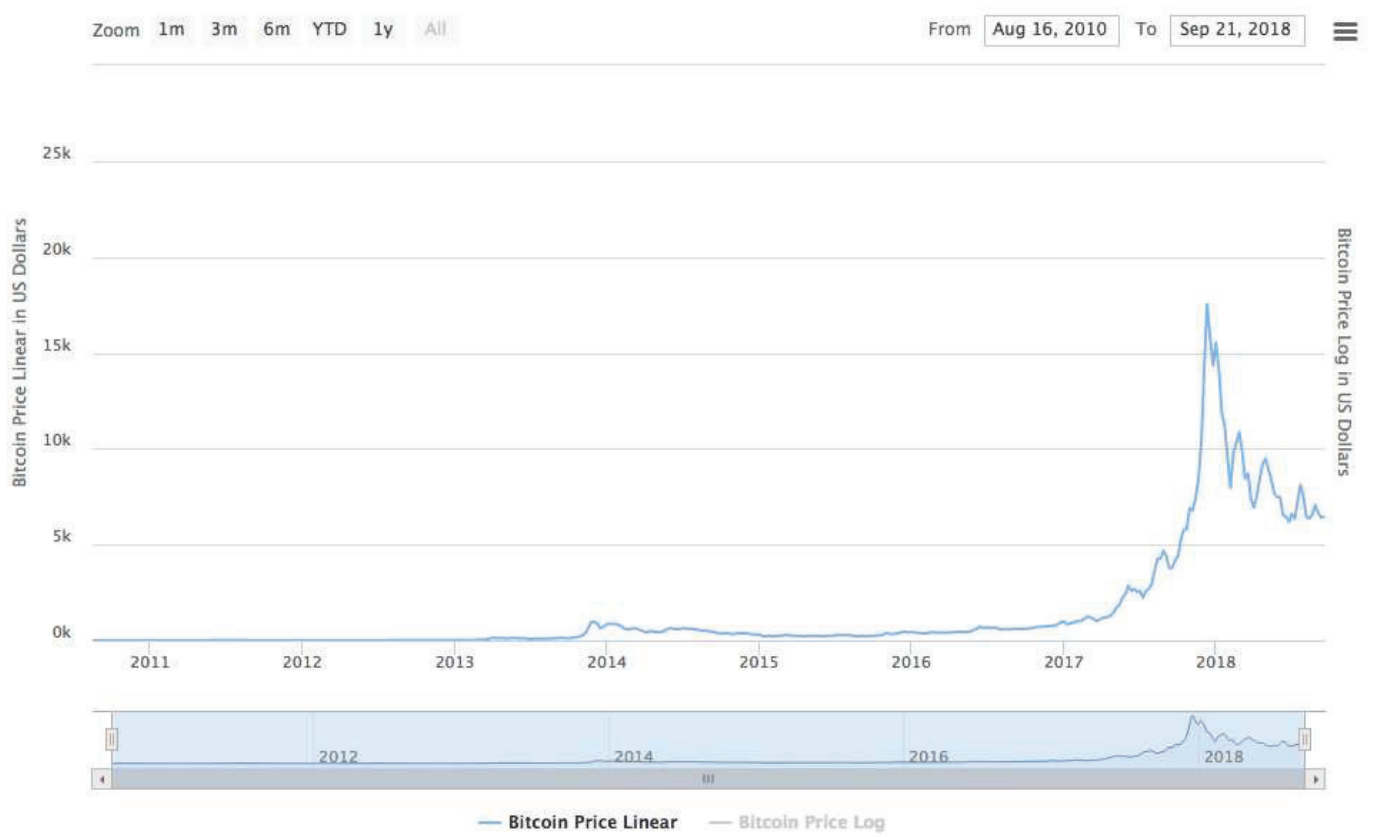

The coin value of Bitcoin soared, allowing some people to see business opportunities, build or expand trading platforms, trying to attract more people to join and earn transaction fees. It is precisely because when more and more players have joined, the value of Bitcoin has begun to rise. 
Table 1: Stages of Evolution of Money. Source: personal elaboration.

\begin{tabular}{|c|c|c|c|c|c|}
\hline \multirow{2}{*}{ N. } & \multirow{2}{*}{$\begin{array}{l}\text { Stage and evolution } \\
\text { of Money }\end{array}$} & \multirow{2}{*}{ Origin time } & \multirow{2}{*}{ Physical currency form } & \multicolumn{2}{|l|}{ Character } \\
\hline & & & & Vantage & Disadvantage \\
\hline 1 & Non-Monetary exchange & c. $? 350 \mathrm{BCE}$ & & Freedom for exchange; environmental protection & $\begin{array}{l}\text { Different sizes, difficult to compare; } \\
\text { no equivalent in exchange }\end{array}$ \\
\hline 2 & Commodity Money & Bronze Age & $\begin{array}{l}\text { Furs; skins; salt; rice; wheat; utensils; ceramic; } \\
\text { livestock; weapons, etc. }\end{array}$ & $\begin{array}{l}\text { Has intrinsic value; environmental protection; } \\
\text { perishable; not easy to store }\end{array}$ & Heavy; difficult to carry \\
\hline 3 & Metallic Money & $1000 \mathrm{BCE}-400 \mathrm{CE}$ & Gold; silver; copper, etc. & Has intrinsic value; easily handled, ascertained & $\begin{array}{l}\text { Inconvenient; dangerous to carry; pollution; } \\
\text { uneven texture; difficult to segment }\end{array}$ \\
\hline 4 & Paper Money & $400-1450$ & Banknotes & $\begin{array}{l}\text { Doesn't has intrinsic value; issued, regulated } \\
\text { and controlled by the central bank }\end{array}$ & $\begin{array}{l}\text { Doesn't has intrinsic value; pollution; } \\
\text { has time limit and geographical restrictions }\end{array}$ \\
\hline 5 & Credit \& Electronic Money & $1450-2008$ & Cheques; credit cards and debit cards & Convenient, smart & Doesn't has intrinsic value; pollution \\
\hline 6 & Digital Money & After 2008 & Bitcoin; ripple; stellar; litecoin; ETH, etc. & Uses cryptography to seek to ensure trust and fungibility & $\begin{array}{c}\text { Doesn't has intrinsic value; pollution; } \\
\text { Instability supported by technology platforms }\end{array}$ \\
\hline 7 & Energy Money & Near future & Solarcoin & Has intrinsic value; convenient; environmental protection & Doesn't need to carry \\
\hline
\end{tabular}

From the process of currency development and its characteristics, as every currency has an important role in each period of economic development, it is important to remember that fiat money is only as good as the Organization that issues it because of the inexistence of an intrinsic value. As a matter of fact, if the entity defaults, the currency will become worthless.

The depreciation of the inflationary currency has led many people to start not to believe in the traditional financial system.

As a matter of fact, we think there is a problem with the financial system, and this problem will cause the depreciation of the money in your hands.

Moreover, in international transactions, the high exchange fee makes transactions of the virtual currency faster. In the past, it was realized in order to avoid the depreciation of the banknotes in the hands.

Virtual currency, after all, it is still money. For this reason, there is the problem of depreciation when a country has too much debt; or because of the responsibilities of the central bank's monetary valuation; or because of the economic growth and central bank policies; or when speculators quickly lose confidence in a country's economic or monetary policy and generate a currency crisis; or the special case of the dollar as a reserve currency, etc.

Energy refers to various resources that can be directly obtained or obtained through processing and conversion. There are many categories including primary energy and electricity such as coal, crude oil, natural gas, coaled methane, hydro, nuclear, wind, solar, geothermal, and biomass; secondary energy sources such as heat and refined oil, as well as other new and renewable energy sources. Bioenergy (also known as biomass) uses organic matter (such as plants) as a fuel to generate energy through technologies such as gas collection, gasification (solidification of gases), combustion and digestion (wet waste only). Biomass energy is also a valuable renewable energy source, as long as it is properly implemented, but it depends on how biomass fuel is produced.

Hydrogen, wind, solar, ocean energy, biomass energy and nuclear fusion energy, the way of new energy, is just a part of the multi-step advancement of energy utilization. Neglected, the engine with great potential or the principle of work and the innovation of ideas are the first direction of energy development in the future.

The conversion of energy includes kinetic energy, potential energy, thermal energy, and light energy, etc. 
Figure 2: Top 3 Producing Countries by Resource Worldwide.

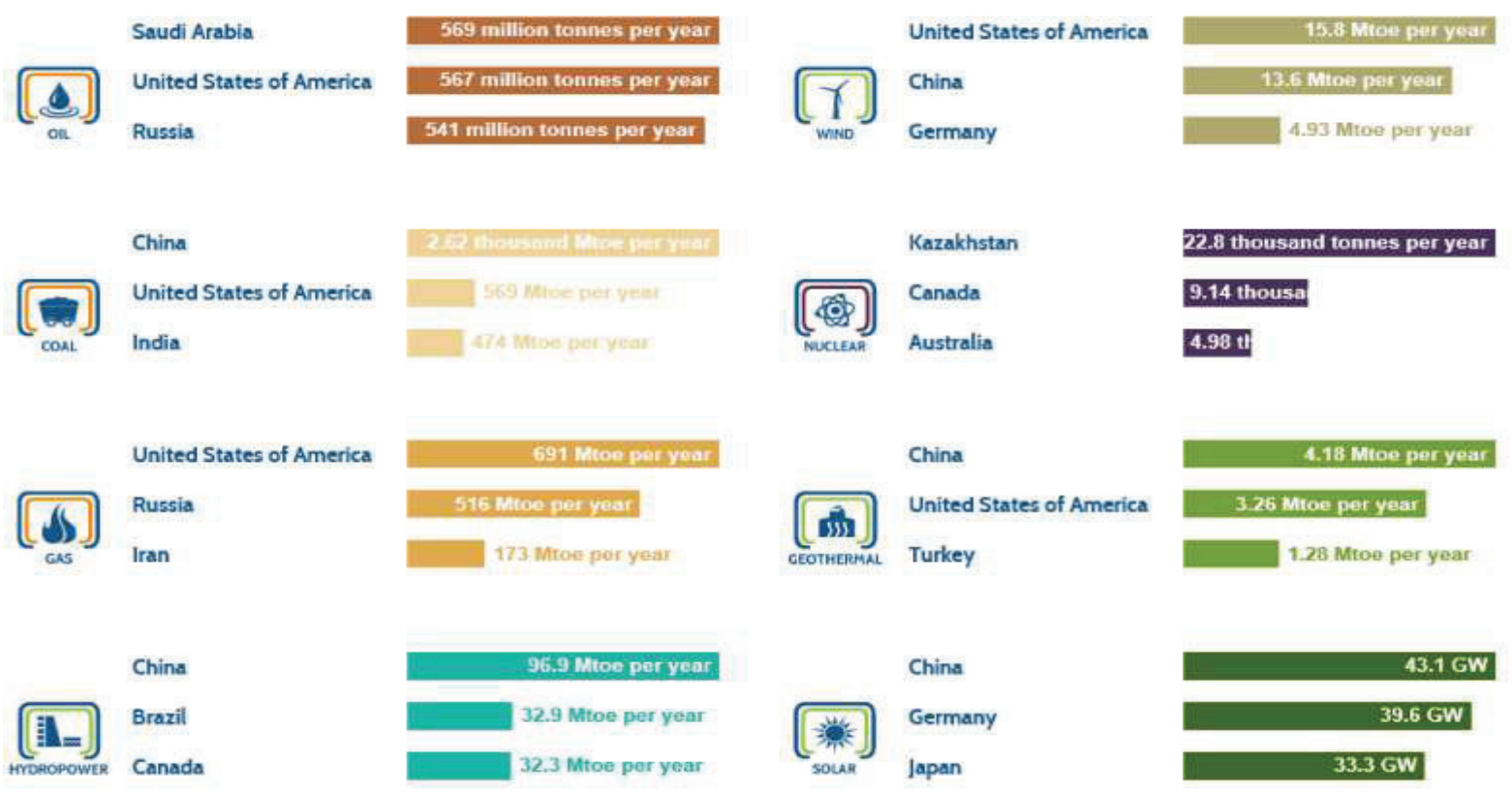

Source: https://www.worldenergy.org/data/resources/

Table 2: Some forms of energy (that an object or system can have as a measurable property).

\begin{tabular}{|c|c|c|c|}
\hline N. & $\begin{array}{l}\text { Type of energy } \\
\text { (from) }\end{array}$ & $\begin{array}{l}\text { Transformation } \\
\text { (to) }\end{array}$ & Description \\
\hline 1 & Mechanical & 14 & the sum of macroscopic translational and rotational kinetic and potential energies \\
\hline 2 & Eletric & 3 & potential energy due to or stored in electric fields \\
\hline 3 & Magnetic & 2 & potential energy due to or stored in magnetic fields \\
\hline 4 & Gravitational & $2,14,15$ & potential energy due to or stored in gravitational fields \\
\hline 5 & Chemical & 2 & potential energy due to chemical bonds \\
\hline 6 & Ionization & 2 & potential energy that binds an electron to its atom or molecule \\
\hline 7 & Nuclear & 3,15 & $\begin{array}{c}\text { potential energy that binds nucleons to form the atomic nucleus (and nuclear } \\
\text { reactions) }\end{array}$ \\
\hline 8 & Chormodynamic & 14 & potential energy that binds quarks to form hadrons \\
\hline 9 & Elastic & 14 & $\begin{array}{c}\text { potential energy due to the deformation of a material (or its container) exhibiting a } \\
\text { restorative force }\end{array}$ \\
\hline 10 & $\begin{array}{l}\text { Mechanical } \\
\text { wave }\end{array}$ & 14 & $\begin{array}{l}\text { kinetic and potential energy in an elastic material due to a propagated deformational } \\
\text { wave }\end{array}$ \\
\hline 11 & Sound wave & 14 & $\begin{array}{l}\text { kinetic and potential energy in a fluid due to a sound propagated wave (a particular } \\
\text { form of mechanical wave) }\end{array}$ \\
\hline 12 & Radiant & 2,14 & $\begin{array}{c}\text { potential energy stored in the fields of propagated by electromagnetic radiation, } \\
\text { including light }\end{array}$ \\
\hline 13 & Rest & 15 & potential energy due to an object's rest mass \\
\hline 14 & Kinetic & 5 & the energy that it possesses due to its motion \\
\hline 15 & Thermal & 2 & $\begin{array}{c}\text { kinetic energy of the microscopic motion of particles, a form of disordered } \\
\text { equivalent of mechanical energy }\end{array}$ \\
\hline
\end{tabular}

Source: personal elaboration. 
Why we say there is the possibility for the energy currency to become the development target of the future currency. Not only because its uniform measurement but also because its good stability characteristics.

As we all know is that Bitcoin, as one of the most important electronic currencies, is not issued by the central bank, but issued by the information service provider. The issuer exists outside the monetary system, which is not conducive to centralized management of the country, and there is a risk of losing control. Finance is not entirely a virtual economy.

This trend has also been asserted by one of relevant scholars, Brett Scott.

"There are good reasons to consider energy as a potential currency, explains Brett Scott, who calls himself Alternate Finance Explorer and author of the book "The Heretic's Guide to Global Finance: Hacking the Future of Money:" Ideally a currency should be anchored to something people always need, so energy-based currencies are interesting because certificates are a necessary asset everywhere. " ... "However radical a hypothesis may appear, energy-based currencies are not completely new and unprecedented. Launched in 2014, SolarCoin (SS) is one of the first examples, and is now adopted in around 21 countries. It provides what is described as a social protocol that supports the exchange of value, where SS1 represents 1 MWh of solar energy production. SolarCoin currently claims to be the world's largest locallybased solar-based remuneration program, valued at over $\$ 12$ billion"... "We are entering an era in which energy is power, literally; a world in which energy is the new currency"

From the current stage, the energy industry is a capital-intensive industry, and financial support plays a vital role in its development. In the process of interactive development of energy finance, the development of the energy industry has promoted innovation and cooperation in the financial industry, as well as the demand for diversified financial services.

The special needs of energy development strategies for financial services have made energy finance face many issues such as support for energy industry development, financial service methods, financial products and even the entire financial market for energy industry innovation.

Both the financial system and the energy industry need innovation, and the combination of the two achieves this goal. If the future integrates energy into the financial system and becomes a means of payment, it will not only eliminate the risk of currency depreciation via global unified measurement unit, unimpeded flow and easily exchange as a general equivalent, but also optimize energy saving and utilization to achieve environmental protection, ultimately to realize the original nature of human return to nature.

\section{LETTERATURE REVIEW}

Before the introduction of the Bitcoin system (BTC), there had already been several attempts to create a secure digital currency and, at the same time, to allow the protection of anonymity of users and to be able to operate without a central authority.

Already in 1998, Wei Dai had conceived "b-money", a virtual currency that had already foreseen a payment system linked to a virtual ledger comparable to the blockchain of the BTC system, but less complex and, therefore, less secure in confirming the transactions. 
In 2005, Nick Szabo presented "bit-gold", from a technical point of view very similar to bitcoins, but with serious fundamental economic defects: there had not been a precise amount of virtual money to distribute and, above all, the speed at which to distribute it; anyone who had procured a powerful enough computer could have produced an almost unlimited quantity, thus eliminating its value.

Undoubtedly, Bitcoin is partly based on the fundamental characteristics of these predecessors, but has been able to stand out for greater efficiency in combining the structural elements of the whole system through the link between blockchain and mining, as will be illustrated below.

Even the hundreds of cryptocurrencies were born after the success of Bitcoin in recent years, they have never detached too much from the fundamental aspects of the BTC system.

As a matter of fact, although the concept of electronic currency dates back to the late 1980s, Bitcoin, launched in 2009 by pseudonymous (and still unidentified) developer Satoshi Nakamoto, it is the first successful decentralized cryptocurrency.

To sum up, a cryptocurrency is a virtual coinage system that functions much like a standard currency, enabling users to provide virtual payment for goods and services without a central trusted authority.

In order for an asset to be used as a currency in commercial transactions, it must possess the following fundamental requirements attached to money:

1. Be a valid unit of account;

2. Guarantee a reserve of value;

3. Being an efficient means of exchange.

Undoubtedly, Bitcoin represented a real revolution with respect to the forms of money adopted in the course of history.

As a unit of account, bitcoins have characteristics superior to the money currently used. Generally, currencies having legal tender are divided into cents, according to a decimal system, while the cryptocurrency can be divided into satoshi, each of which corresponds to 0.00000001 bitcoins, ensuring unparalleled accuracy, regardless of the type of currency in which or from which it is converted: a considerable advantage offered by the dematerialization of money.

In this way, even if the value of bitcoins were to increase enormously in the future, microtransactions and micropayments in any currency will still be technically possible in the coming years.

Cryptocurrencies are based on the transmission of digital information, using cryptographic methods to guarantee unique and legitimate transactions.

Bitcoin has taken the digital money market a step further by decentralizing the currency and freeing it from hierarchical structures of power. Instead, individuals and businesses electronically process coins on a peer-to-peer network.

It caught wide attention in early 2011 and several altcoins appeared - a generic name for all other post-Bitcoin cryptocurrencies. 
Litecoin was released in the autumn of 2011, obtaining a modest success and enjoying the highest cryptocurrency market cap after Bitcoin until it was exceeded by Ripple on October 4, 2014. Litecoin changed the Bitcoin protocol, increasing the speed of transactions with the idea that it would be more suitable for daily transactions.

Ripple, launched in 2013, introduced a completely unique model compared to that used by Bitcoin and currently maintains the second highest market capitalization of about $\$ 255$ million.

Another noteworthy coin in the evolutionary chain of cryptocurrency, Peercoin, employs revolutionary technological development to secure and sustain its currency.

Peercoin combines the PoW technology used by Bitcoin and Litecoin along with its proof-ofstake (PoS) mechanism to use a hybrid network security mechanism.

More recently, NuShares / NuBits emerged, introduced in August 2014, which are based on a double currency model almost entirely separated from the single currency model used by previous crypto-currencies.

At the time this paper was written, the cryptocurrency industry consisted of over 2080 coins with different user bases and trade volumes.

Due to the high volatility, the market capitalization of the cryptocurrency sector changes drastically, but at the time of writing this paper has just finished \$181,142 billion, with Bitcoin accounting for about $53 \%$ of market capitalization.

The cryptocurrency market has evolved in an irregular way and at an unprecedented speed during its short duration. Since the release of the pioneering anarchist cryptocurrency, Bitcoin, to the public in January 2009, over 2080 cryptocurrencies have been developed, most with only a minimum of success.

Research on industry is still scarce. Most of it is individually focused on Bitcoin rather than on a more diversified spread of cryptocurrencies and is constantly outperformed by developments in the fluid industry, including new currencies, technological progress and increased government regulation of markets.

Although the fluidity of the industry, of course, is a challenge to research, a thorough evaluation of the cryptocurrency industry is required.

Following the success of Bitcoin, hundreds of alternative cryptocurrencies have been created, many of which have tried to solve the technical problems of bitcoins (above all the very high generation costs) proposing structural changes, while many others are in fact simple copies of bitcoin and the original blockchain system.

The proliferation of these new currencies is due in most cases to the hope of easy profits, if the price of the new currency should increase dramatically as it has occurred with the Bitcoins, especially considering that this kind of activity does not require nor relevant initial investments or even special protocols and regulations to follow. 
Furthermore, the original Bitcoin system is completely open source. On 15th November 2018, according to the Coin-MarketCap, Bitcoin holds about 53\% of the cryptocurrency market and is by far the most famous and important.

Among the cryptocurrencies that have spread the most in recent years there are Litecoin, GeistGeld, SolidCoin and BBQcoin; these currencies are also called altcoins, as we have already said before.

Going more in detail, Litecoin, created in 2011 by Charles Lee, was conceived as an updated and perfected version of the Bitcoin system: the new algorithm introduced allows any user to extract the currency, with far more efficient energy consumption. In this way, moreover, the whole system prevents that only users who have a very high computational power can profit from the Litecoin system; the algorithm also allows to shorten the transactions. Furthermore, the final and total money supply will be equal to 84 million units (and therefore the quadruple with respect to the Bitcoins); however the offer, even if higher, is however limited and this will not allow Litecoin to avoid the deflationary problems linked also to the Bitcoin system. Also the Feathercoin, introduced in 2013 by Peter Bushnell, guarantees an offer of currency that, even if equal to four times that of Litecoin, is still limited.

Cryptocurrencies like Peercoin and Novacoin, on the other hand, propose a completely different approach with the proof-to-stake system as an alternative to proof-to-work: when a cryptocurrency unit is successfully created, those who have solved the cryptographic puzzle are not rewarded. But, a sort of "dividend" is distributed to all the units already in circulation, rewarding those who hold the most. In fact, even in the extraction of these cryptocurrencies 15 are the users with more powerful hardware to get the best results. An interesting case is that of Ethereum Project (founded in 2014), which proposes to use blockchain technology even in areas other than online payments and completely disconnected from the economic sphere, for example to ensure the security of votes made via internet. Such potential applications, although the entire project is not yet complete, have attracted the attention of IT giants such as Microsoft and Intel, but also of companies such as Airbus and Toyota. The price of Ethereum's currency, Ether, has grown by $2700 \%$ from January to May 2017, even exceeding the very high bitcoin growth rates in the same period (see Kharpal 2017).

To these are added the so-called "digital currencies", including Liberty Reserve, WebMoney, Perfect Money and CashU, but they are not based on cryptography and must be purchased using an intermediary (even if most of them try to guarantee to the user the complete anonymity), renouncing to the attractiveness of the principle of decentralization.

With the sole exception of Litecoin and Ethereum, however, all these currencies are still scarcely used in real transactions and the Bitcoins undoubtedly still enjoy the advantages of the first-mover, which in a context like this could prove more relevant than technological superiority. Certainly the vast majority of cryptocurrencies will not be used in the future and the growth of the last few years of the entire sector is mainly due to massive speculation.

Many users, in fact, buy dozens of different cryptocurrencies without any intention of using them as a medium of exchange, waiting to be able to resell them at higher prices in the future. It is not for a very long time that money is the cash as we understand it today. In Japan, for example, rice was the unit of account of the great fiefs until the second half of the nineteenth century. In Iceland, at the end of the same century, the price of each commodity was established 
on the basis of the equivalent of dried fish. And going even further back in the centuries, the commodity currency was represented by cattle, shells, obsidian, salt, tea and pieces of fabric.

Today, however, we have cash. But that of money is a world that is anything but static: the very popular checks are becoming an historical find, while electronic payments are now part of everyday life, through credit cards or even smartphones.

At the base of all these digital transactions, however, are always the national currencies: the euro, the dollar, the pound, the yen ... And if instead we began to use energy as a global currency? This is the proposal / provocation by Brett Scott, author of The Heretic's Guide to Global Finance: Hacking the Future of Money.

There are good reasons to consider energy as a potential currency, explains Brett Scott who calls himself an Alternative Finance Explorer.

Ideally, a currency should be anchored to something people always need, so currencies based on energy are interesting because the certificates represent a necessary good everywhere.

Alternatively we could think of these currencies as a financing instrument - the possibility of collecting funding for a renewable energy project through the issue of representative certificates of energy that will be produced in the future (in this sense, the currencies based on energy to a certain extent resemble equity certificates).

However radical a hypothesis may appear, energy-based currencies are not completely new and unprecedented.

There are already embryonic examples of energy as a currency. There is, for example, the SolarCoin cryptocurrency (whose symbol is §), launched in 2014 to stimulate the production of solar electricity globally.

SolarCoin $(\S)$ is one of the first examples, and today it is adopted in around 21 countries.

It provides what is described as a social protocol that supports the exchange of value, where $\S 1$ represents $1 \mathrm{MWh}$ of solar energy production. SolarCoin currently claims to be the world's largest locally-based solar-based remuneration program, valued at over $\$ 12$ billion.

SolarCoin, a project supported by an open community, was created in 2014 by its own founders and some volunteers who set up the SolarCoin Foundation. SolarCoin Foundation rewards solar energy producers with blockchain-based digital tokens at a rate of 1 SolarCoin (SLR) for $1 \mathrm{MWh}$ of solar energy produced.

Based on Blockchain technology - the same as BitCoin - it provides a social protocol that supports a value exchange, in which $1 \S$ is equivalent to $1 \mathrm{MWh}$ of solar energy produced.

The SolarCoin project uses the advantages of the blockchain to support the development of solar energy on the planet. SolarCoin is a digital asset and a currency intended to support the transition from an economy focused on fossil fuels to an economy supported by solar energy.

The Solarcoin Foundation rewards solar energy producers through digital tokens based on blockchain technology at the price of 1 SolarCoin (SLR) per MWh of solar energy produced. 
SolarCoin is an additional premium recognized free of charge and independent of any other incentives that solar plant owners might be entitled to receive (such as government incentives, incentive tariffs, green certificates, tax credits, $\mathrm{CO} 2$ reduction credits, etc.). Anyone producing solar energy, helping to avoid $\mathrm{CO} 2$ emissions, can receive a reward in SolarCoin.

The SolarCoin project is global, decentralized and independent of any government. Solarcoin is similar to other cryptocurrency like Bitcoin, but unlike these, SolarCoin 'binds' the distribution of money to a real economic activity of public utility: the production of verifiable solar energy.

Different but with a very similar objective is the initiative of the British start-up ZAPP, which aims to create and place on the world market an app that renumber consumers for the storage and use of renewable energy. Its operation is based on the Zapp Points, or points that customers can earn by charging their electrical appliances synchronizing to the production of renewable energy. The Zapp Points earned by the customer can then be exchanged for goods and services or converted into cash.

There is then another initiative that brings the concept of energy closer to that of currency to be used concretely for the physical development of the real world. It has its base of operations in New York, more precisely in Brooklyn, on President Street: here the startup Transactive Grid has launched a pioneering project that uses a microrete powered by solar energy, boasting a technology based on the cloud and the Internet of Things. The New York experiment aims to allow peer-to-peer energy exchanges: if a President Street building generates excess solar energy, it is automatically made available to other houses. And all this happens without the use of cash, through the secure platform for Ethereum transactions, also based on Blockchain.

As you can understand from these initiatives, in short, the hypothesis of the energy used as a currency is not so radical: something is already moving, in a global perspective for which energy is increasingly synonymous with power (and then, in some ways, even cash).

Another type of cryptocurrency based on energy is represented by Energy Efficiency Coin (EECoin).

Energy Efficiency Coin is a blockchain asset class designed to have a positive ecological impact and to track a weighted aggregate of real world renewable energy markets, giving token holders the opportunity to vote on which renewable resource markets will be included in the weighted average price. Energy efficiency coin is a pegged asset in which the price is linked to a weighted super index of the constituent green equity and bond indices, where keeping the price index peg becomes the incentive trading strategy and the revenue stream for the company of token shareholding, ensuring future financing for capital management and the development of technical platforms.

Additional type of coin based on renewable energy is represented by Electronic Energy Coin (E2C) that is a trading block platform for green technology.

E2C is built according to the ERC-20 token standard, which is used by the famous Ethereum system. This makes E2C compatible with most of the cryptocurrency standards and protocols on the market. 
In E2C, we look at an ecological and sustainable future. Our project will be based on an energy revolution, a fairer distribution and better control over the use of energy.

Conventionally, the energy market is dominated by a few people. This entails high costs for ordinary people, such as owners of private companies.

All these types of crypto currencies are a testimony to how there is a link between the world of crypto currencies with the energy sector and therefore with the financial world.

However, it should be noted that most of the cryptocurrencies based on energy sector assets are limited only to certain energy sources, such as, in particular, renewable energy sources.

Our intention is to present a new interpretation for this topic, giving the possibility to identify a standard method that can be used for the whole energy sector.

\section{METHOD}

The Kinetic energy is the energy that an object has due to its motion and its expression is as follows, with the meaning of half mass times velocity squared:

$$
E_{k}=\frac{1}{2} m v^{2}
$$

Where, if direction and speed direction are the same:

$$
\begin{gathered}
E_{k}, \text { is the energy of a mass, } m, \text { in motion, } v^{2} \\
E_{k}=\text { Kinetic energy } \\
m=\text { mass }(\mathrm{kg}) \\
v=\text { velocity }(\mathrm{m} / \mathrm{sec})
\end{gathered}
$$

Kinetic energy is a scalar, also a state quantity. The expression is as follows:

$$
\text { Unit: Joule (J) } 1 \mathrm{~kg} \cdot \mathrm{m}^{2} / \mathrm{s}^{2}=1 \mathrm{~J}
$$

After combined the above two formulas, we have:

$$
E_{k}=1 \mathrm{~kg} \mathrm{~m} / \mathrm{sec}^{2}=1 \text { Joules }
$$

\section{DISCUSSION AND CONCLUSION}

The international unit of energy is joule (or abbreviated as J). We can consider it as the unit of energy money which is the energy that has intrinsic value. This new kind of energy cryptocurrency differs from existing solutions as it is based globally on the energy macrocategory and not on a sub-sector. This element is what makes this solution new and represents a strong point compared to existing solutions.

While intrinsic value means that the commodity has value even if it is not used as money. As energy there are production, exchange, reserves, distribution and consumption. 
As there is neither energy nor mass can be destroyed; rather, both remain constant during any process, there is no energy depreciation, on the contrary only energy appreciation. In addition, using energy as a currency, there is no need for printing or casting and applying the exchange rate, so there is no corresponding pollution.

Even better, it will contribute to relieve the environmental problem in the current stage and in a practical and effective way to meet the challenges of global environmental degradation.

Energy issuer and reserve agency - International Energy Bank (IEB) which will be given support and capacity for combination through IMF (International Monetary Fund) and IEA (International Energy Agency) to realize the unique currency form - Energy and its unified currency symbol "Joule (J)".

\section{REFERENCES}

[1] Scott, B. (2013). The heretic's guide to global finance: hacking the future of money. London: Pluto Press.

[2] Junfeng, Li. (2010) Energy and financial crisis, Science Press, 2010.7, isbn:7030282647, 9787030282644 , Bei Jing.

[3] Halliday, D., Resnick, R., \& Walker, J. (2010). Fundamentals of Physics, Chapters 33-37. John Wiley \& Sons.

[4] Del Mar, A. (1885). A History of money in ancient countries from the earliest times to the present. G. Bell.

[5] Richards, R. D. (1929). The Early History of Banking in England. London: King.

[6] Weatherford, J. (2009). The history of money. Crown Business.

[7] Crypto-Currency Market Capitalizations. [Online]. http://coinmarketcap.com/

[8] Satoshi Nakamoto. (2008) Bitcoin: A Peer-to-Peer Electronic Cash System. [Online]. https://bitcoin.org/bitcoin.pdf

[9] David Schwartz, Noah Youngs, and Arthur Britto, "The Ripple Protocol Consensus Algorithm," Ripple Labs Inc,.

[10] Scott Nadal Sunny King. (2012, August) PPCoin: Peer-to-Peer Crypto-Currency with Proof-of-Stake. [Online].

[11] Aron, J. (2014). What's wrong with Bitcoin?.

[12] Vigna, P., \& Casey, M. J. (2016). The age of cryptocurrency: how bitcoin and the blockchain are challenging the global economic order. Macmillan.

[13] Lee, Charles. "Litecoin." (2011).

[14] Fanning, K., \& Centers, D. P. (2016). Blockchain and its coming impact on financial services. Journal of Corporate Accounting \& Finance, 27(5), 53-57.

[15] Antonopoulos, A. M. (2014). Mastering Bitcoin: unlocking digital cryptocurrencies. "O’Reilly Media, Inc.".

[16] Dispenza, J., Garcia, C., \& Molecke, R. (2017). Energy Efficiency Coin (EECoin) A Blockchain Asset Class Pegged to Renewable Energy Markets. 